Originalien

Monatsschr Kinderheilkd 2021 • 169:46-51 https://doi.org/10.1007/s00112-020-01067-8 Angenommen: 8. September 2020

Online publiziert: 19 . November 2020

(c) Springer Medizin Verlag GmbH, ein Teil von Springer Nature 2020

\section{Redaktion}

B. Koletzko, München

T. Lücke, Bochum

E. Mayatepek, Düsseldorf

N. Wagner, Aachen

S. Wirth, Wuppertal

F. Zepp, Mainz

\begin{abstract}
Nach derzeitigem Kenntnisstand erkranken Kinder seltener als Erwachsene an COVID-19. Zudem verläuft die Erkrankung bei ihnen meist milder als bei erwachsenen Patienten. Bisher gibt es nur wenige Daten zu der Prävalenz von SARS-CoV-2-Infektionen in einer prospektiv abgestrichenen pädiatrischen Kohorte. Im vorliegenden Beitrag stellen wir die Daten aus einer Kohorte von 2192 Kindern und Jugendlichen, welche retrospektiv ausgewertet wurden, vor. Diese Daten sind gerade im Hinblick auf die begonnenen Schulöffnungen relevant, weil sie Aussagen über die Infektionsprävalenz in bestimmten Altersgruppen ermöglichen. Unsere Daten ermöglichen Abschätzungen zu Übertragung und Verbreitung des Virus bei Kindern.
\end{abstract}

\section{Einleitung}

Seit dem ersten bekannten Auftreten des neuen Coronavirus SARS-CoV-2 im Dezember 2019 in der chinesischen Stadt Wuhan hat sich das Virus weltweit ausgebreitet. In Deutschland sind bisher (Stand 18.06.2020) 187.764 SARS-CoV2-Infektionen nachgewiesen. Hiervon verstarben 8856 Personen im Zusam-

C. Hünseler und L. T. Weber teilen sich die Letztautorenschaft.

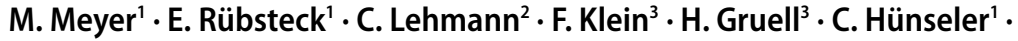
L. T. Weber ${ }^{1}$

'Medizinische Fakultät und Uniklinik Köln, Klinik und Poliklinik für Kinder- und Jugendmedizin, Universität zu Köln, Köln, Deutschland

${ }^{2}$ Medizinische Fakultät und Uniklinik Köln, Innere Medizin I (Onkologie, Hämatologie, Klinische Infektiologie, Klinische Immunologie, Hämostaseologie, Internistische Intensivmedizin), Universität zu Köln, Köln, Deutschland

${ }^{3}$ Medizinische Fakultät und Uniklinik Köln, Institut für Virologie, Universität zu Köln, Köln, Deutschland

\title{
Prävalenz von SARS-CoV-2 bei Kindern in einer Kohorte von 2192 Patienten
}

menhang mit der Infektion (4,7\%) [1]. Kinder scheinen insgesamt seltener an COVID-19 zu erkranken. Gemäß den größten Kohorten in China, Italien und den USA [2-4] sind ca. 1-2\% der gemeldeten positiven Patienten unter 18 Jahre alt. In Deutschland sind aktuell ungefähr $13.001(6,9 \%)$ der Infizierten unter 19 Jahre alt [1]. Kinder erkranken nicht nur seltener, die Verläufe der COVID19-Erkrankung sind meist milde oder asymptomatisch [5, 6]. Laut einer Studie aus China verlaufen nur ca. 5\% der Fälle schwer [7]. In Deutschland mussten $13 \%$ der erfassten 150 hospitalisierten Kinder intensivmedizinisch behandelt werden. Ein Patient verstarb. Die genauen Hintergründe hierzu sind noch nicht bekannt [8]. Ziel der vorliegenden Arbeit ist es, einen Überblick über die Infektionsprävalenz bei 1261 abgestrichenen Kindern und Jugendlichen $\mathrm{zu}$ geben.

\section{Methodik}

Im Rahmen der aktuellen SARS-CoV-2Pandemie wurde in der 11. Kalenderwoche (KW) 2020 (09.03-15.03.2020) auf dem Gelände der Uniklinik Köln in enger Zusammenarbeit mit der Klinischen Infektiologie der Medizinischen Klinik I und dem Institut für Virologie ein Infektionsschutzzentrum für Erwachsene und Kinder aufgebaut. Dort wurde die Durchführung sämtlicher Abstriche bei Ver- dachtspersonen (Verdachtsfall entsprechend den Kriterien des Robert Koch-Instituts in fortlaufender Anpassung) bzw. zum Ausschluss einer SARS-CoV-2-Infektion (Ausschlussfall, z.B. vor elektiver stationärer Aufnahme, durchgeführt ab 13. KW) konzentriert. Im Rahmen der Vorstellung wurden Symptome und mögliche Kontaktpersonen erfragt und dokumentiert. Zudem wurden an der Klinik für Kinder- und Jugendmedizin der Uniklinik Köln im Zuge des Infektionsschutzes ebenfalls seit der 11. KW alle akut über die Notaufnahme aufgenommenen Patienten abgestrichen. Ab Ende März (13. KW) musste auch für alle $\mathrm{Pa}$ tienten, die eine geplante Intervention oder weiterführende Diagnostik in der Klinik erhalten bzw. stationär aufgenommen werden sollten, ein max. $72 \mathrm{~h}$ altes Abstrichergebnis vorliegen (Ausschlussfälle).

Der Abstrich wurde als oro- und nasopharyngealer Abstrich vom Fachpersonal durchgeführt. Die Probe wurde umgehend in unsere Virologie weitergeleitet. Dort erfolgte der Virusnachweis mittels Polymerase-Kettenreaktion (PCR). Bei einer Subgruppe von Patienten (z. B. bei besonders kranken Patienten oder bei Patienten, wo die Therapie nicht ansprach) wurden in einer Multiplex-PCR zusätzliche folgende Erreger untersucht: Influenza A, Influenza B, Parainfluenza, Adenovirus, Metapneumovirus, 
Tab. 1 Übersicht der demografischen $\mathrm{Da}$ ten der Kohorte $(n=2192)$

Gesamtzahl ( $n=2192)$

\begin{tabular}{ll}
\hline Alter (Median) & $\begin{array}{l}\text { 5,5 Jahre } \\
\text { (Min. } 0 \text { Jahre; Max. 28,8) }\end{array}$ \\
\hline $\begin{array}{l}\text { Geschlecht } \\
\text { (männlich) }\end{array}$ & $53,3 \%(n=1169)$ \\
\hline $\begin{array}{l}\text { Verdachtsfälle } \\
\text { Ausschlussfälle }\end{array}$ & $41,2 \%(n=903)$ \\
\hline Risikopatienten & $23,0 \%(n=1289)$ \\
\hline
\end{tabular}

Coronavirus HKU1/NL63/OC43, RSV, Rhinovirus, Bocavirus und Enterovirus.

Statistische Auswertung: Zwischen dem 13.03.2020 und dem 18.06.2020 (11.-25. KW) wurden alle Kinder und Jugendlichen, welche in der Uniklinik Köln einen Abstrich auf SARS-CoV-2 erhielten, erfasst. Die Daten wurden auf standardisierten Anamnesebogen gesammelt und retrospektiv mittels einer Excel ${ }^{\circledR}$-Datenbank (Microsoft, Redmond, Washington, USA) tabellarisch erfasst.

Folgende anonymisierte Daten/Informationen wurden erhoben: Geschlecht, Alter, Vorstellungsgrund (Verdachtsfall, Ausschlussfall), Zugehörigkeit zu einer Risikogruppe (chronische kardiologische, onkologische, pneumologische, nephrologische oder endokrinologische Vorerkrankung) und Abstrichergebnisse auf weitere respiratorische Erreger, falls erfolgt.

Die deskriptive Darstellung der Ergebnisse erfolgte bei numerischen Variablen als Angabe des Mittelwertes und der Standardabweichung bzw. des Medians mit Angabe des Minimal- und Maximalwerts bei fehlender Normalverteilung der Stichprobe. Kategorische Variablen wurden als prozentualer Wert des zugrunde liegenden Kollektivs angegeben.

Wir unterteilten die Patienten für die Auswertung in zwei Gruppen. Als Verdachtsfälle wurden alle Kinder und Jugendliche bezeichnet, welche entweder Kontakt $\mathrm{zu}$ einer nachweislich SARS-CoV-2-infizierten Person hatten und/oder symptomatisch (u.a. Fieber, Husten und Schnupfen) waren, unabhängig, ob sie über die Notaufnahme aufgenommen wurden oder im Infektionsschutzzentrum gesehen wurden. Alle nichtsymptomatischen Kinder und

Tab. 2 Vergleich der Verdachtsfälle vs. der Ausschlussfälle

\begin{tabular}{|c|c|c|}
\hline & $\begin{array}{l}\text { Verdachtsfälle } \\
(n=903)\end{array}$ & $\begin{array}{l}\text { Ausschlussfälle } \\
(n=1289)\end{array}$ \\
\hline Alter (Median) & $\begin{array}{l}\text { 6,7 Jahre } \\
\text { (Min. 0,2 Jahre; Max. 18,7 Jahre) }\end{array}$ & $\begin{array}{l}\text { 4,8 Jahre } \\
\text { (Min. } 0 \text { Jahre; Max. 19,6 Jahre) }\end{array}$ \\
\hline Geschlecht (männlich) & $52,9 \%(n=478)$ & $53,6 \%(n=691)$ \\
\hline $\begin{array}{l}\text { Kontakt zu einem SARS-CoV-2- } \\
\text { positiven Patienten }\end{array}$ & $26,1 \%(n=236)$ & $0 \%(n=0)$ \\
\hline Symptome & $88,9 \%(n=803)$ & $0 \%(n=0)$ \\
\hline \multicolumn{3}{|l|}{ Symptome nach Häufigkeit } \\
\hline Trockener Husten & $56,5 \%(n=454)$ & - \\
\hline Fieber & $49,7 \%(n=399)$ & - \\
\hline Schnupfen & $36,1 \%(n=290)$ & - \\
\hline Halsschmerzen & $34,6 \%(n=278)$ & - \\
\hline Kopfschmerzen & $27,3 \%(n=219)$ & - \\
\hline Gastrointestinale Symptome & $14,6 \%(n=117)$ & - \\
\hline Gliederschmerzen & $13,1 \%(n=105)$ & - \\
\hline Geschmacksstörungen & $2,9 \%(n=23)$ & - \\
\hline Geruchsstörungen & $0,1 \%(n=1)$ & - \\
\hline
\end{tabular}

Jugendliche, die vor einer stationären Aufnahme oder einer elektiven ambulanten Vorstellung einen Abstrich aufSARSCoV-2 erhielten, wurden in der Gruppe Ausschlussfälle zusammengefasst.

\section{Ergebnisse}

Im genannten Zeitraum wurden die Daten von insgesamt 2192 Patienten erfasst. Hiervon erfolgten 1849 Testungen im Infektionsschutzzentrum sowie 343 Testungen bei ungeplanten stationären Aufnahmen über die Notaufnahme.

Es gab 903 Verdachtsfälle $(41,2 \%)$ und 1289 Ausschlussfälle (58,8\%). Das mediane Alter der Gesamtkohorte betrug 5,5 (Min. 0, Max. 28,8) Jahre Das jüngste Kind wurde einen Tag nach Geburt getestet, da es aus einer externen Geburtsklinik in unsere Klinik verlegt wurde. Der älteste erfasste Patient war 28,8 Jahre alt. Insgesamt wurden 43 Patienten über 18 Jahre erfasst, die aufgrund ihrer chronischen Grunderkrankung in Spezialambulanzen der Kinderklinik betreut werden (•Tab. 1).

Die Gruppe der Verdachtsfälle wird in - Tab. 2 mit der Gruppe der Ausschlussfälle verglichen.

Insgesamt wurden 37 Kinder und Jugendliche $(1,7 \%)$ positiv getestet. Hiervon waren 17 weiblich und 20 männlich. Das mediane Alter betrug 9,8 Jahre (Min.
0,2 Jahre, Max. 19,5 Jahre) Jahre. Bei 31/37 Patienten lag ein Kontakt zu einer gesichert SARS-CoV-2-positiven Person vor $(83,8 \%) .6 / 37$ Patienten $(16,2 \%)$ hatten keinen wissentlichen Kontakt zu einer SARS-CoV-2-positiven Person. Fünfdieser 6 Patienten ohne wissentlichen Kontakt hatten als Verdachtsfälle klinische Symptome. Die einzige als Ausschlussfall positiv getestete 19-jährige Patientin war eine Patientin mit bekannter zystischer Fibrose. Den Vergleich zwischen SARS-CoV-2-positiv getesteten Patienten mit denen ohne Virusnachweis zeigt - Tab. 3.

Insgesamt wurden 3 Patienten wegen COVID-19 stationär betreut, davon 2 zur kardiorespiratorischen Überwachung aufgrund ihres jungen Alters (8 Wochen und 6 Monate). Die beiden Säuglinge wurden bei leichten klinischen Verläufen rein symptomatisch behandelt. Bei der 6 Monate alten Patientin konnte zudem eine Koinfektion mit Adenovirus nachgewiesen werden. Ein 11,9 Jahre alter Patient wurde zur neurologischen Überwachung nach Sturz aufgenommen und bei Aufnahme positiv getestet. Bei bekanntem positivem SARS-CoV-2-Abstrich der Eltern galt er als Verdachtsfall und befand sich bereits in Quarantäne. Er zeigte keine Symptome. Alle stationären Kinder wurden nach 2 Tagen entlassen. 
Im beschriebenen Zeitraum konnten wir keinen Fall eines Hyperinflammationssyndroms im Zusammenhang mit einer COVID-19-Erkrankung beobachten.

Den Verlauf durchgeführter Tests auf SARS-CoV-2 sowie den Anteil positiv getesteter Kinder und Jugendlicher zwischen der 11. und 25. KW 2020 zeigt - Abb. 1.

Unter den 2192 getesteten Patienten waren 505 Risikopatienten (23,0\%). Drei Risikopatienten $(0,6 \%)$ wurden positiv aufSARS-CoV-2 getestet. Ein Patient mit bekanntem Diabetes mellitus Typ 1 sowie eine Patientin mit bekannter zystischer Fibrose zeigten asymptomatische Verläufe. Ein weiterer Patient mit komplexem Herzvitium zeigte lediglich leichte Symptome einer Infektion der oberen Atemwege und konnte ambulant geführt werden.

Wie bereits oben beschrieben, konnte bei einer COVID-19-Patientin eine Koinfektion mit Adenovirus nachgewiesen werden. Koinfektionen mit anderen respiratorischen Erregern zeigten sich in unserer Kohorte nicht. Insgesamt 71 der 2192 Patienten wurden neben SARS-CoV-2 auch auf RSV, Influenza und teils weitere respiratorische Viren getestet. 57/71 wurden lediglich auf RSV- und Influenza A bzw. B getestet. Es zeigten sich 10 RSV-Infektionen, 4 Influenza-A-Infektionen, jeweils 2 Infektionen mit Boca- und Corona-NL63Viren, eine Influenza-B-Infektion, eine Adenovirusinfektion, eine Infektion mit Metapneumo- und Rhinoviren und eine Infektion mit Boca- und Rhinovirus. Seit Ende März gab es in unserer Kohorte keinen Influenzanachweis mehr.

\section{Diskussion}

Im Rahmen der SARS-CoV-2-Epidemie wird aktuell weltweit versucht, möglichst viele Informationen $\mathrm{zu}$ dem neuartigen Coronavirus SARS-CoV-2 zu sammeln. Auch wenn Kinder in den meisten Fällen nur milde oder sogar asymptomatische Verläufe zeigen, kommt ihnen eine besondere Rolle zu, da sie durch den Kindergarten- bzw. Schulbesuch täglich viele soziale Kontakte haben und Abstandsund Hygieneregelungen oftmals schwer

Monatsschr Kinderheilkd 2021 · 169:46-51 https://doi.org/10.1007/s00112-020-01067-8

(c) Springer Medizin Verlag GmbH, ein Teil von Springer Nature 2020

\section{Meyer · E. Rübsteck · C. Lehmann · F. Klein · H. Gruell · C. Hünseler · L. T. Weber Prävalenz von SARS-CoV-2 bei Kindern in einer Kohorte von 2192 Patienten}

\section{Zusammenfassung}

Hintergrund. In Deutschland sind aktuell (Stand 18.06.2020) 187.764 SARS-CoV-2-Infektionen gemeldet. 6,9\% der Infizierten sind dabei unter 19 Jahre. Es gibt erste Hinweise darauf, dass Kinder häufig asymptomatisch sind und einen milderen Verlauf zeigen.

Ziel der Arbeit. Ziel dieser Arbeit war es, eine Aussage bezüglich der Infektionsprävalenz in dieser speziellen Patientengruppe zu ermöglichen.

Material und Methoden. Im Rahmen der SARS-CoV-2-Pandemie wurden zwischen dem 13.03.2020 und dem 18.06.2020 alle Kinder und Jugendlichen, welche entweder zum Ausschluss einer SARS-CoV-2-Infektion oder als Verdachtsfall einen Abstrich auf SARSCoV-2 erhielten, erfasst. Die Daten wurden auf standardisierten Anamnesebogen gesammelt und retrospektiv anonymisiert ausgewertet. Ergebnisse. In dem Zeitraum wurden 2192 Kinder und Jugendliche auf SARS-CoV-2 abgestrichen. 37 Patienten wurden positiv getestet (1,7\%). 36/37 waren Verdachtsfälle, und 28/37 waren symptomatisch. Führende Symptome waren trockener Husten, Schnupfen und Fieber. Drei Kinder mussten stationär betreut werden. Keines zeigte einen schweren Verlauf. Unter den Getesteten waren 505 Patienten, die aufgrund ihrer chronischen Grunderkrankung als Risikopatienten eingestuft wurden, von denen $3(0,6 \%)$ einen positiven Nachweis mit asymptomatischem bzw. mildem Verlauf hatten.

Schlussfolgerung. Hiermit können die ersten Daten bestätigt werden, wonach Kinder und Jugendliche häufig asymptomatische oder klinisch milde Infektions- bzw. Erkrankungsverläufe zeigen. Zudem fanden wir keinen Hinweis für eine hohe Dunkelziffer für SARS-CoV-2-Infektionen in dieser regionalen pädiatrischen Kohorte.

Schlüsselwörter

Deutschland · COVID-19 · Dunkelziffer .

Symptome $\cdot$ Risikopatienten

\section{Prevalence of SARS-CoV-2 in children from a cohort of 2192 patients}

\section{Abstract}

Background. As of 18 June 2020 a total of 187,764 severe acute respiratory syndrome coronavirus 2 (SARS-CoV-2) infections were reported in Germany and of these $6.9 \%$ were under the age of 19 years. There were initial indications that children are often asymptomatic and show a milder clinical course.

Objective. The aim of this study was to gain information on the prevalence of SARS-CoV-2 infections in a pediatric cohort.

Material and methods. Between 13 March and 18 June 2020 all children from whom a smear for SARS-CoV-2 was taken either to rule out an infection or as a suspected case were included. Data were collected on standardized patient record sheets. The analysis of data was anonymized and retrospective.

Results. During the given period 2192 children were investigated and 37 patients tested positive (1.7\%) for SARS-CoV-2. Of these $36 / 37$ were suspected cases and $28 / 37$ were symptomatic. The leading symptoms were dry cough, runny nose and fever and three children had to be hospitalized. None showed a difficult course of the disease. Among those tested 505 were patients at risk due to an underlying chronic disease, 3 of whom (0.6\%) were tested positive with an asymptomatic or mild course.

Conclusion. We can confirm the first data showing that children and adolescents often have an asymptomatic or mild clinical course of infection or disease. We found no evidence of a high grey area of SARS-CoV-2 infections in this regional pediatric cohort.

Keywords

Germany · COVID-19 · Dark figures .

Symptoms $\cdot$ Patients at risk 


\begin{tabular}{|c|c|c|}
\hline & $\begin{array}{l}\text { SARS-CoV-2-positiv } \\
(n=37)\end{array}$ & $\begin{array}{l}\text { SARS-CoV-2-negativ } \\
(n=2155)\end{array}$ \\
\hline Alter (Median) & $\begin{array}{l}\text { 9,8 Jahre } \\
\text { (Min. 0,2 Jahre; Max. 19,5 Jahre) }\end{array}$ & $\begin{array}{l}\text { 5,5 Jahre } \\
\text { (Min. } 0 \text { Jahre; Max. 28,8 Jahre) }\end{array}$ \\
\hline Geschlecht (männlich) & $54,1 \%(n=20)$ & $53,3 \%(n=1149)$ \\
\hline $\begin{array}{l}\text { Kontakt zu einem SARS-CoV-2- } \\
\text { positiven Patienten }\end{array}$ & $83,8 \%(n=31)$ & $9,5 \%(n=205)$ \\
\hline \multicolumn{3}{|l|}{ Altersgruppen } \\
\hline $0-<3$ Jahre & $24,3 \%(n=9)$ & $32,4 \%(n=698)$ \\
\hline $3-<6$ Jahre & $18,9 \%(n=7)$ & $20,5 \%(n=441)$ \\
\hline $6-<12$ Jahre & $18,9 \%(n=7)$ & $24,2 \%(n=521)$ \\
\hline 12-18 Jahre & $35,1 \%(n=13)$ & $21,0 \%(n=453)$ \\
\hline$>18$ Jahre & $2,7 \%(n=1)$ & $1,9 \%(n=42)$ \\
\hline Symptomatisch & $75,7 \%(n=28)$ & $36,0 \%(n=775)$ \\
\hline \multicolumn{3}{|l|}{ Symptome nach Häufigkeit } \\
\hline Trockener Husten & $60,7 \%(n=17)$ & $56,4 \%(n=437)$ \\
\hline Schnupfen & $53,6 \%(n=15)$ & $35,5 \%(n=275)$ \\
\hline Fieber & $42,9 \%(n=12)$ & $49,9 \%(n=387)$ \\
\hline Kopfschmerzen & $35,7 \%(n=10)$ & $27,0 \%(n=209)$ \\
\hline Halsschmerzen & $32,1 \%(n=9)$ & $34,7 \%(n=269)$ \\
\hline Gliederschmerzen & $25,0 \%(n=7)$ & $12,6 \%(n=98)$ \\
\hline Gastrointestinale Symptome & $25,0 \%(n=7)$ & $14,2 \%(n=110)$ \\
\hline Geschmacksstörungen & $3,6 \%(n=1)$ & $2,8 \%(n=22)$ \\
\hline Geruchsstörungen & $0 \%(n=0)$ & $0,1 \%(n=1)$ \\
\hline
\end{tabular}

umzusetzen sind. Im Rahmen des Krisenmanagements der Bundesregierung wurden alle Betreuungseinrichtungen zum 16.03.2020 geschlossen. Seit dem 20.04.2020 erfolgt nun in NordrheinWestfalen die langsame und schrittweise Öffnung der Einrichtungen.

Die hier vorgestellte Fallserie bestätigt erste Beobachtungen, wonach Kinder seltener erkranken und mildere Verläufe der COVID-19-Erkrankung zeigen. Die Rate potenziell infektiöser Kinder von $1,7 \%$ liegt in unserem Kollektiv im Zeitraum vom 13.03.2020 bis zum 18.06.2020 niedrig. Jedoch muss bei der Betrachtung unserer Zahlen bedacht werden, dass wir nicht nur Verdachtsfälle getestet haben, sondern 58,8\% der Kinder und Jugendlichen Ausschlussfälle waren. In einer Querschnittsstudie aus Island lag der Anteil positiv getesteter Personen (Kinder und Erwachsene) bei 0,8\%. Bemerkenswert ist hier, dass keines der Kinder unter 10 Jahre alt war [9]. In unserer Kohorte lag der Anteil der positiv auf SARSCOV-2 getesteten Kindern und Jugendlichen bei $0,1 \%$ bei Ausschlussfällen. In der Gruppe der Verdachtsfälle unserer wurden, welche nachweislich Kontakt $\mathrm{zu}$ einer SARS-CoV-2-positiven Person hatten. Im weiteren Verlauf der Pandemie wurde dann empfohlen, dass nur noch Kinder getestet wurden, welche stationär aufgenommen werden mussten und entsprechende Symptome hatten oder einer Risikogruppe angehörten. Hierdurch lassen sich vermutlich die deutlich höheren Fallzahlen erklären [11]. Insgesamt sind die bisher veröffentlichten epidemiologischen Daten aufgrund unterschiedlicher Methodik nur schwer zu vergleichen.

Häufig wurde eine hohe Dunkelziffer (Patienten, welche mit SARS-CoV-2 infiziert, aber nicht identifiziert sind) an infizierten Patienten insbesondere bei Kindern und Jugendlichen vermutet. Dies lässt sich anhand unserer Daten nicht bestätigen. Von den 2192 getesteten Patienten gab es lediglich einen positiven Zufallsbefund. Die Patientin hatte keinen wissentlichen Kontakt zu einer SARSCoV-2-positiven Person und war asymptomatisch. Sie wurde im Rahmen eines Screenings identifiziert. Alle anderen positiv auf SARS-CoV-2 getesteten Patienten hatten entweder nachweislich Kontakt zu einer an COVID-19 erkrankten Person $(83,8 \%)$ und befanden sich daher bereits in häuslicher Quarantäne oder hatten Symptome.

Im Rahmen eines allgemeinen Aufnahmescreenings erwachsener Patienten unseres Zentrums ließen sich im Zeitraum vom 12.04 .2020 bis 18.06 .2020 unter 7211 untersuchten Proben $7 \mathrm{Zu}$ fallsbefunde $(0,1 \%)$ feststellen. Betrachtet man die Kinder ebenfalls zwecks Vergleichbarkeit in dem gleichen Zeitraum, kommt man ebenfalls auf eine Rate von $0,08 \%$ bei den Zufallsbefunden $(1 / 1180)$.

Wir konnten, wie bereits von anderen Kollegen berichtetet [12-14], bei Kindern und Jugendlichen ebenfalls keine erhöhte Infektionsrate unter Risikopatienten bzw. keine schweren klinischen Verläufe feststellen. Die 3 positiv getesteten chronisch kranken Patienten zeigten einen milden bzw. asymptomatischen Verlauf. Jedoch muss auch hier bedacht werden, dass die Gruppe der Risikopatienten mit 505 Patienten klein war, und dass nur 6,9\% $(n=35)$ 


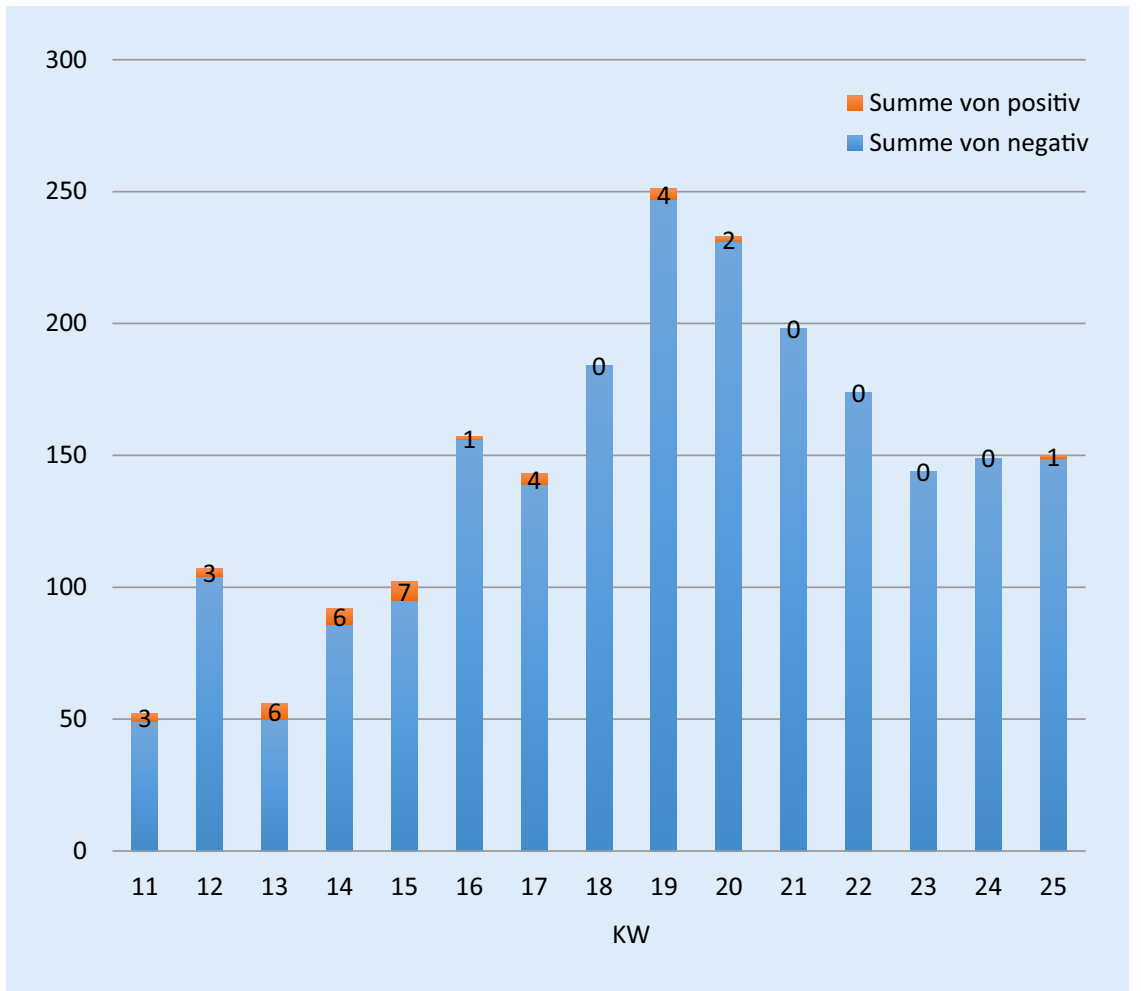

Abb. $1 \Delta$ Häufigkeit der durchgeführten Abstriche und Anteil positiver SARS-CoV-2-Ergebnisse in der 11.-20. KW. KW Kalenderwoche

dieser Patienten symptomatisch waren. Alle anderen chronisch kranken Patienten waren asymptomatisch und wurden im Rahmen des prästationären Screenings getestet. Anzumerken ist, dass die Erfassung des möglichen COVID19 assoziierten Symptoms der Geruchsund Geschmacksstörung in unserer Kohorte lediglich erfragt und nicht konkret getestet wurde. Es ist davon auszugehen, dass lediglich ältere Kinder hierzu eine belastbare Aussage machen konnten.

Schober et al. wiesen darauf hin, dass flächendeckende Schließungen von Betreuungseinrichtungen vermutlich weniger als zuvor angenommen zur Eindämmung der Pandemie beitragen. Daher plädierten sie für eine Öffnung der Einrichtungen, gerade auch im Hinblick auf die psychosozialen Kollateralschäden, welche durch die Schließungen entstehen [15]. Wir sehen insgesamt eine sehr geringe Infektionsprävalenz bei Kindern und Jugendlichen in unserem Kollektiv. Vor allem können wir die Sorge vor einer hohen Dunkelziffer nicht bestätigen. In aller Regel lag bei SARS-CoV-2-Nachweis zuvor ein Pri- noch erhebliche Lockdown-Maßnahmen (inkl. Schulschließungen) vorlagen. Die allgemeine Prävalenz (und vielleicht besonders bei Kindern/Jugendlichen) wurde hierdurch möglicherweise noch zusätzlich beeinflusst. Weitergehende aussagekräftige Erhebungen von Infektionszahlen sind aus diesem Grunde unabdingbar.

\section{Fazit für die Praxis/ Kernaussagen}

- Geringe Infektionsprävalenz bei Kindern und Jugendlichen,

- Symptomatik der SARS-CoV-2Infektion unspezifisch,

- kein Hinweis für hohe Dunkelziffer in pädiatrischer Patientenpopulation,

- keine erhöhte Infektionsrate unter Risikopatienten bzw. keine schweren Verläufe bei Risikopatienten,

- Kinder und Jugendliche zeigen häufig asymptomatische oder klinische milde Infektions- bzw. Erkrankungsverläufe.

\section{Korrespondenzadresse

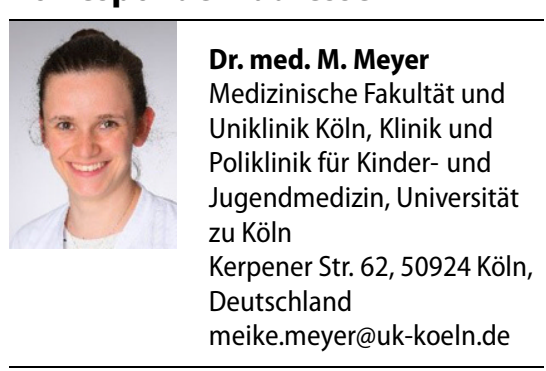
werden, dass im Falle eines negativen PCR-Befunds keine Wiederholung des Rachen-/Nasenabstrichs erfolgte.

Durch den hohen Anteil von Ausschlussfällen (58,8\%) in unserer Kohorte, der potenziell eine Selektion von Patienten bedeutet, bildet unsere Kohorte keinen repräsentativen Querschnitt der Altersgruppe ab. Zudem erfolgten im Großraum Köln neben den Abstrichen in unserem Infektionsschutzzentrum vorübergehend auch Abstriche in zwei weiteren Infektionsschutzzentren und bei niedergelassenen Kollegen. Möglicherweise sind dadurch die leicht erkrankten bzw. asymptomatischen Kinder unterrepräsentiert. Der Einfluss dieser Aktivitäten auf die Zusammensetzung unserer Kohorte ist nicht abschließend beurteilbar, da der jeweilige Umgang mit Verdachts- und Ausschlussfällen sehr heterogen war. Des Weiteren spiegelt unsere Erhebung eine Querschnittssituation des Großraums Köln wider und ist nicht ohne Weiteres auf andere Regionen übertragbar. Zudem wurden die Zahlen größtenteils in einem Zeitraum erhoben, in dem
Einhaltung ethischer Richtlinien

Interessenkonflikt. M. Meyer, C. Lehmann, F. Klein, H. Gruell, C. Hünseler und L. T. Weber geben an, dass kein Interessenkonflikt besteht.

Für diesen Beitrag wurden von den Autoren keine Studien an Menschen oder Tieren durchgeführt. Für die aufgeführten Studien gelten die jeweils dort angegebenen ethischen Richtlinien.

\section{Literatur}

1. https://www.rki.de/DE/Content/InfAZ/N/ Neuartiges_Coronavirus/Situationsberichte/ 2020-06-18-de.pdf?_blob=publicationFile. Zugegriffen: 18. Juni 2020

2. Wu Z, McGoogan JM (2020) Characteristics of and important lessons from the Coronavirus Disease 
2019 (COVID-19) outbreak in China: summary of a report of 72314 cases from the Chinese Center for Disease Control and Prevention. JAMA 323(13):1239-1242

3. Livingston E, Bucher K (2020) Coronavirus Disease 2019 (COVID-19) in Italy. JAMA 323(14):1335

4. Centre for Disease Control Coronavirus Disease 2019 in children-United States, Feburary 12-April 2 2020. https://www.cdc.gov/mmwr/ volumes/69/wr/mm6914e4.htm. Zugegriffen: 18. Juni 2020

5. Chidini G, Villa C, Calderini E, Marchisio P, De Luca D (2020) SARS-CoV-2 infection in a pediatric department in Milan: a logisticrather than a clinical emergency. Pediatr Infect Dis J 39:e79-e80

6. Frentheim A, Stoltenberg C Folkehelseinstituttet/Norwegian institute of public health: MEMO-COVID-19-EPIDEMIC: the role of children in the transmission of SARS-CoV-2 (COVID-19)-a rapid review. https://www.fhi.no/ globalassets/dokumenterfiler/rapporter/2020/ the-role-of-children-in-the-transmission-ofsars-cov-2-report-2020.pdf. Zugegriffen: 17. Mai 2020

7. Dong $Y$ et al (2020) Epidemiology of COVID-19 among children in china. Pediatrics. https://doi. org/10.1542/peds.2020-0702

8. Armann JP et al (2020) Hospital admission in children and adolescents with COVID-19-early results from a national survey conducted by the German Society for Pediatric Infectious Diseases (DGPI).Dtsch Arztebl Int 117:373-374

9. Gudbjartsson DF, Helgason A, Jonsson $\mathrm{H}$, Magnusson OT et al (2020) Spread of SARS-CoV-2 in the Icelandic population. N Engl J Med. https://doi. org/10.1056/NEJMoa2006100

10. https://www.rki.de/DE/Content/Infekt/EpidBull/ Archiv/2020/Ausgaben/25_20.pdf?_blob= publicationFile. Zugegriffen: 18. Juni 2020

11. Tagarro A, Epalza C, Santos M, Sanz-Santaeufemia FJ, Otheo E, Moraleda C et al (2020) Screening and severity of coronavirus disease 2019 (COVID19 ) in children in Madrid, Spain. JAMA Pediatr. https://doi.org/10.1001/jamapediatrics.2020. 1346

12. Sainati L, Biffi A (2020) How we deal with the COVID-19 epidemic in an Italian paediatric oncohaematology clinic located in a region with a high density of cases. Br JHaematol 189:640-642

13. Boulad F, Kamboj M, Bouvier N, Mauguen A, Kung AL (2020) COVID-19 in children with cancer in New York City. JAMA Oncol. https://doi.org/10. 1001/jamaoncol.2020.2028

14. Marlais M, Wlodkowski T, Vivarelli M, Pape $L$, Tönshoff B, Schaefer F, Tullus K (2020) The severity of COVID-19 in children on immunosuppressive medication. Lancet Child Adolesc Health. https:// doi.org/10.1016/S2352-4642(20)30145-0

15. Schober T, Rack-Hoch A, Kern A, von Both U, Hübner J (2020) Coronakrise: Kinder haben das Recht auf Bildung. Dtsch Arztebl 117(19):A990-4

\section{Jahre DPV-Register}

Wo stehen wir heute in der Versorgung von Kindern und Jugendlichen mit Diabetes?

Seit nunmehr 25 Jahren besteht mit dem DPV-Register für Kinder, Jugendliche und junge Erwachsene eine bundesweite Initiative zur Qualitätssicherung, die individuelle Patientendaten wie Geschlecht, Manifestationsalter und Diabetestyp multizentrisch erfasst. „Durch diese Datensammlung von inzwischen über 92.000 Kindern und Jugendlichen konnten wir über die Jahre insgesamt eine Verbesserung in der Stoffwechseleinstellung der Patientinnen und Patienten feststellen“, bilanziert DDG Experte Professor Dr. med. Reinhard Holl, Vorsitzender der AG Diabetologie Baden-Württemberg e.V.. „Darüber hinaus liefert es uns einmalige Erkenntnisse zu seltenen Diabetesformen wie dem Neugeborenen-Diabetes sowie Diabetes als Medikamenten-Nebenwirkung oder aktuell zu COVID-19-Auswirkungen auf Diabetespatienten."

Insgesamt liegt in Deutschland eine gute medizinische Versorgungslage vor und die Therapieergebnisse haben sich in den vergangenen Jahrzehnten deutlich verbessert. Eine wichtige Entwicklung der vergangenen Jahre war der Einzug neuer Technologien wie Insulinpumpen und die kontinuierliche Glukosemessung in die Praxis. Bei immer jüngeren Patienten wurden diese erfolgreich eingesetzt: Während für das Behandlungsjahr 2015 noch bei 932 Kindern und Jugendlichen mit Diabetes Typ 1 eine sensorunterstützte Pumpentherapie (SUP) entweder mittels kontinuierlicher Glukosemessung (CGM) oder Flash Glucose Monitoring (FGM) dokumentiert war, waren es drei Jahre später bereits 10.180 Patientinnen und Patienten.

„Aber nicht alles wird besser: Die Zahl gefährlicher Stoffwechselentgleisungen mit diabetischer Ketoazidose hat leider nicht abgenommen", erklärt Holl, Leiter der Arbeitsgruppe Computergestütztes Qualitätsmanagement in der Medizin im epidemiologischen Institut der Universität Ulm. Noch immer besteht eine diabetische Ketoazidose bei jedem fünften Patienten unter 20 Jahren bei Diagnosestellung des Typ-1-Diabetes. "Höchste Priorität hat daher die Früherkennung: Vermehrtes Trinken, häufiges Wasserlassen und Gewichtsabnahme sind die ersten Anzeichen einer Diabeteserkrankung", erklärt Professor Dr. med. Andreas Neu, Vizepräsident der DDG. Sind diese Symptome vorhanden, sei ein umgehender Arztbesuch zwingend notwendig um schwere Stoffwechselentgleisungen, die lebensbedrohlich werden können, zu verhindern, so der Kommissarische Ärztliche Direktor an der Klinik für Kinder- und Jugendmedizin am Universitätsklinikum Tübingen.

Hinzu kommt die steigende Zahl von Typ-2Diabetes-Patienten in dieser Altersgruppe. "Viele Kinder und Jugendliche in Deutschland haben heute Übergewicht oder sind adipös. Der Typ-2-Diabetes, der früher als „Altersdiabetes" bezeichnet wurde, wird heute auch bei manchen schwer adipösen Minderjährigen diagnostiziert", führt Holl aus. In Deutschland sind derzeit laut DPV-Register und anderen Datenquellen rund 1.000 Jugendliche betroffen, darunter knapp 70 Prozent Mädchen. 30 Prozent der Betroffenen haben einen Migrationshintergrund.

Auch regionale Unterschiede sind durch die DPV-Datenerfassung abbildbar. Unter den 16 Bundesländern schwankt beispielsweise die Stoffwechseleinstellung bei Kindern und Jugendlichen mit Typ-1-Diabetes zwischen 7,5 Prozent und 8,4 Prozent. Schwere Unterzuckerungen (Hypoglykämien) treten je nach Bundesland bis zu 3,5-mal häufiger auf.

Am DPV-Register beteiligen sich derzeit 283 pädiatrische und 205 internistische Einrichtungen. 440 Zentren sind aus Deutschland, 43 aus Österreich, vier aus der Schweiz und eines aus Luxemburg. Inzwischen wurden etwa 300 Publikationen zur DPV-Initiative veröffentlicht. Insgesamt sind mittlerweile knapp 650.000 Menschen mit Diabetes im DPV-Register erfasst.

\section{Quelle: www.deutsche-diabetes-} gesellschaft.de 